\title{
Numerical evaluation of ABS parts fabricated by fused deposition modeling and vapor smoothing
}

\author{
Sung-Uk Zhang* \\ Electro Ceramic Center, Dept. of Automotive engineering, Dong-Eui University, Busan, 47340, South Korea
}

\begin{tabular}{l} 
A R T I C L E I N F O \\
\hline Article history: \\
Received: 26 October, 2017 \\
Accepted: 01 December, 2017 \\
Online: 10 December, 2017
\end{tabular}

Keywords:

Additive manufacturing

Fused deposition modeling

$A B S$

Vapor smoothing

Finite element analysis

Response surface analysis

\begin{abstract}
A B S T R A C T
The automotive industry has focused to use polymer materials in order to increase energy efficiency. So, the industry pays attention to use $3 D$ printing technologies using several polymers. Among several 3D printer technologies, fused deposition modeling (FDM) is one of the popular $3 D$ printing technologies due to an inexpensive extrusion machine and multimaterial printing. FDM could use thermoplastics such as ABS, PLA, ULTEM so on. However, it has a problem related to the post-processing because FDM has relatively poor layer resolution. In this study, the mechanical properties of ABS parts fabricated by FDM were measured. The ABS parts were divided into one with vapor smoothing process and the other without the vapor smoothing process which is one of the post-processing methods. Using dynamic mechanical analysis (DMA) and dilatometer, temperature-dependent storage modulus and CTE for ABS specimens were measured. Based on the measured thermo-mechanical properties of ABS parts, finite element analysis was performed for an automotive bumper made of ABS. Moreover, response surface methodology was applied to study relationships among design parameters of thickness of the bumper, ambient temperature, and application of the vapor smoothing process. In result, a design guideline for a ABS product could be provided without time-consuming experiments
\end{abstract}

\section{Introduction}

The automotive industry has tried to increase energy efficiency for vehicles. In order to achieve the goal, one of ways is to reduce vehicle weight. If weight of the vehicle increases by $10 \%$, fuel economy also increases by about $7 \%$ [1]. Therefore, researchers in the automotive industry have focused to use materials lighter than steel. One of the substitute materials is polymer. Currently, about $15 \%$ to $20 \%$ of total weight of a vehicle is in charge of polymer materials. The polymer materials are widely used for automobile parts including front ends, bumpers, fascia systems, grills, fenders, intake manifolds, engine mounts, door structures, panels, door impact beams, bonnet panels, under hood parts, pedal boxes, body structures, steering columns and so on [1]. Among several polymers, acrylonitrile butadiene styrene (ABS) is applied for bumpers, seats, dashboard, interior trim, exterior trim, and lighting and so on. This study focuses on ABS because fused deposition modeling (FDM), which is one of additive manufacturing techniques, could use $\mathrm{ABS}$ to make 3D printed structures.

Recently, additive manufacturing also known as 3D printing is considered as the key driver of the fourth industrial revolution.

"Corresponding Author: Sung-Uk Zhang, 82-51-890-1645, zsunguk@deu.ac.kr
Compared to the conventional subtractive manufacturing such as turning, cutting, milling and grinding, 3D printing has unique advantages in design flexibility, personal fabrication, cost of geometry complexity, dimensional accuracy, time and cost efficiency in production run and so on [2]. Based on these merits, rapid development has been made in the several industrial areas such as aviation parts, manufacturing, and medical science [3]. However, the 3D printing has demerits of, scalability, trade-off between building and layer resolution, cost for mass manufacturing, material heterogeneity, need of anchor and support material, requirement for post-processing and so on [2]. So many researchers have solved the problems for the next generation of the $3 \mathrm{D}$ printing technique [2].

This study focuses on the post processing technique for the additive manufacturing. Additive manufacturing techniques have been classified into seven categories by ASTM international, which are shown in Figure.1.

Among them, FDM belongs to a category of material extrusion, which is an inexpensive and very popular 3D printing technique [2]. But FDM sometimes struggles with poor surface finish so that the post processing technique is necessary. The poor surface finish occurred by stair stepping effect could be treated by 


\section{S.U. Zhang / Advances in Science, Technology and Engineering Systems Journal Vol. 2, No. 6, 157-161 (2017)}

polishing process such as sanding and vapor smoothing. The sanding may not be possible for tiny or complex geometries. On the other hand, the vapor smoothing could be effective for the complex shapes fabricated by 3D printing as shown in Figure. 2.

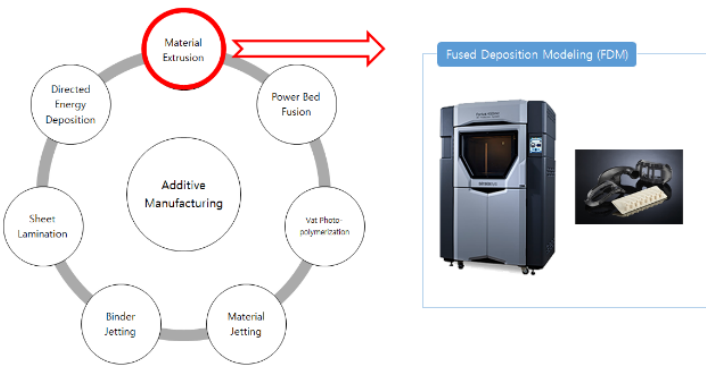

Figure.1. Seven categories for AM technologies

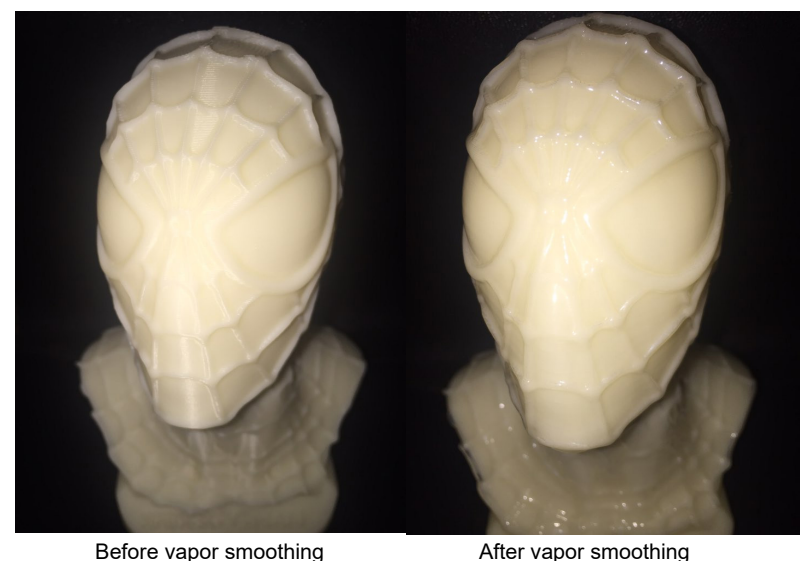

Figure.2. Vapor smoothing before and after

Many researchers have studied the vapor smoothing process and its effect in the mechanical properties. Kuo and Mao [4] developed an acetone-vapor smoothing system for ABS parts fabricated by FDM. They showed that surface roughness of the ABS parts could be significantly improved by using their system. Garg et al. [5] observed mechanical properties and surface roughness according to building parameters and vapor smoothing process for ABS specimens fabricated by FDM. In order to obtain the mechanical properties, they used ultimate testing machine (UTM). Singh et al. [6] studied vapor smoothing process for FDM parts. They had tried to improve the vapor smoothing process in term of surface roughness and dimensional accuracy by using DOE technique. Singh et al. [7] optimized building parameters of FDM and control parameters of vapor smoothing processes for hip joint as a biomedical application. They used Taguchi's method and response surface methodology to obtain the optimum level of process parameters. Cantrell et al. [8] measured mechanical properties of $3 \mathrm{D}$ printed $\mathrm{ABS}$ and $\mathrm{PC}$ parts with varying raster and building orientation of additive manufacturing. Gao et al. [9] investigates the impact of the vapor smoothing for 3D printed ABS parts in terms of the strength and elongation. They measured anisotropic mechanical properties of the ABS parts and showed that the material properties are affected by the length of process time for the vapor smoothing. Belter and Dollar [10] suggested a technique to strengthen 3D printed parts fabricated by FDM. They tried to remove voids in the printed parts by filling them with highstrength resins. They performed three-point bending test to obtain mechanical properties. Melenka et al. [11] evaluated mechanical properties and dimensional accuracy for 3D printed PLA parts fabricated by FDM. They used DOE technique to establish a relationship between building parameters and mechanical properties of the $3 \mathrm{D}$ printed parts.

In this study, temperature-dependent mechanical properties of 3D printed ABS parts fabricated by FDM and the acetone-vapor smoothing are measured by using dynamic mechanical analyzer (DMA) and dilatometer. Based on the measured data, finite element analysis and response surface methodology are performed in order to generate a design guideline for an automotive bumper made of ABS.

\section{Methodology}

The second section has four subsections. The first subsection includes geometry information of specimen for DMA and dilatometry. The second briefly explains about the vapor smoothing process. The third subsection describes about information measured by DMA and dilatometry. The last subsection introduces about finite element analysis and response surface methodology for an automotive bumper made of ABS.

\subsection{Specimen fabrication for DMA and Dilatometry}

Specimens for DMA and dilatometry were fabricated by using Fortus 3D printer which is based on Stratasys FDM technology. In modeler setup of Stratasys's software, building parameters were controlled, which are part interior style, visible surface style, and support style be set to sparse-low density, being enhanced, and being smart. The specimen for DMA has $27.8 \mathrm{~mm}$ length with 6.5 $\mathrm{mm}$ width and $2.0 \mathrm{~mm}$ thickness and the size of specimen for dilatometry is $6 \mathrm{~mm}$ by $6 \mathrm{~mm}$ by $12 \mathrm{~mm}$ as shown in Figure. 3 .

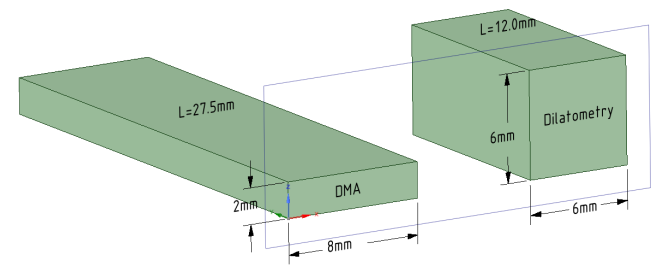

Figure.3. Geometry of the specimen for DMA and Dilatometer

\subsection{Vapor smoothing process}

Vapor smoothing process lets ABS plastic dissolve in acetone. So the secondary bonds between the ABS polymer chains is broken so that external layers of ABS 3D printed parts are softening. In result, this technique makes the surface finish of $3 \mathrm{D}$ printed parts be improved. A simple process for the vapor smoothing is shown in Figure. 4.

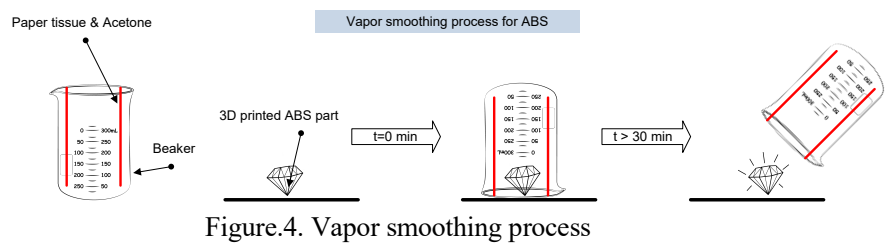

First of all, dust and debris from 3D printed ABS parts are removed. Small amount of acetone is poured on paper tissues. The tissues are attached inside of a beaker. Then, the 3D printed parts are covered with the beaker. Then, allow parts to be inside of the beaker for about 30 minutes. In this paper, amount of acetone was controlled from $5 \mathrm{ml}$ to $15 \mathrm{ml}$ in order to observe a relationship between the amount of acetone and mechanical properties of the 3D printed parts with the vapor smoothing. 


\subsection{Dynamic mechanical analyzer and Dilatometry}

Dynamic Mechanical Analyzer (DMA), which known as DMA, is a powerful tool to characterize mechanical properties of a material as a function of temperature, time, frequency, stress, and so on. Q800 DMA was used, which is manufactured by TA instruments. Temperature-dependent storage modulus of ABS 3D printed parts could be observed by using DMA. As an applied mechanical load of DMA is a sinusoidal or oscillating force, complex modulus of $\mathrm{E}^{*}$ could be obtained for polymer materials such as ABS, PLA, PC, and ULTEM, which consists of the storage modulus E' (real part) and the loss modulus E" (imaginary part) as follow

$$
\begin{aligned}
& \mathbf{E}^{*}=E^{\prime}+\mathbf{i} E^{\prime \prime} \\
& \tan \delta=E^{\prime \prime} / E^{\prime}
\end{aligned}
$$

where, $\tan \delta$ is the ratio of loss modulus to storage modulus, which is an indicator of the energy lost. Figure.5 graphically shows temperature dependent storage modulus and $\tan \delta$ measured by using DMA. In the graph of $\tan \delta$, the peak of $\tan \delta$ indicates the glass transition temperature which means a midpoint between the glassy and rubbery states of a polymer.

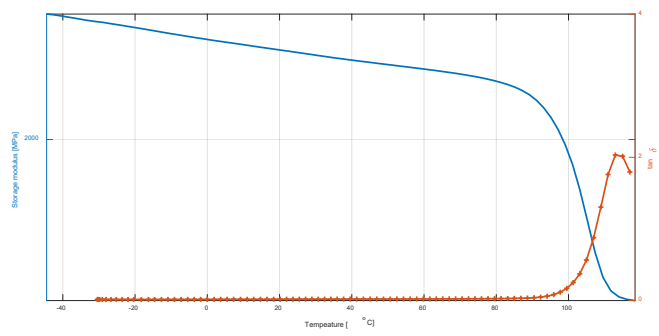

Figure.5.Temperature dependent storage modulus and $\tan \delta$ obtained by using DMA

Moreover, a dilatometer is used to measure temperature dependent CTE of the polymer materials, which is an instrument to measure volume changes occurred by a physical or chemical event. In this study, the physical event is temperature change. The thermal expansion is expressed as

$$
\alpha=\frac{1}{V}\left(\frac{\partial V}{\partial T}\right)
$$

where, $\alpha$ is a coefficient of thermal expansion (CTE), $\mathrm{V}$ is a volume, and T is a temperature. We used DIL-402C manufactured by NETZSCH. Using the dilatometry, we could obtain temperature dependent CTEs for ABS 3D printed parts.

\subsection{Finite element analysis and response surface methodology for a $3 D$ printed bumper made of $A B S$}

A portion of a bumper is modeled and structurally evaluated by using finite element analysis as shown in Figure. 6 and Figure. 7. Fig 6 shows the geometry of the automotive bumper. Fig 7 graphically shows fixed boundary conditions, load boundary conditions, and body temperature condition on the geometry. In Figure. 7, the symbols of A, B and C mean boundary conditions of fixed support, pressures, thermal conditions.

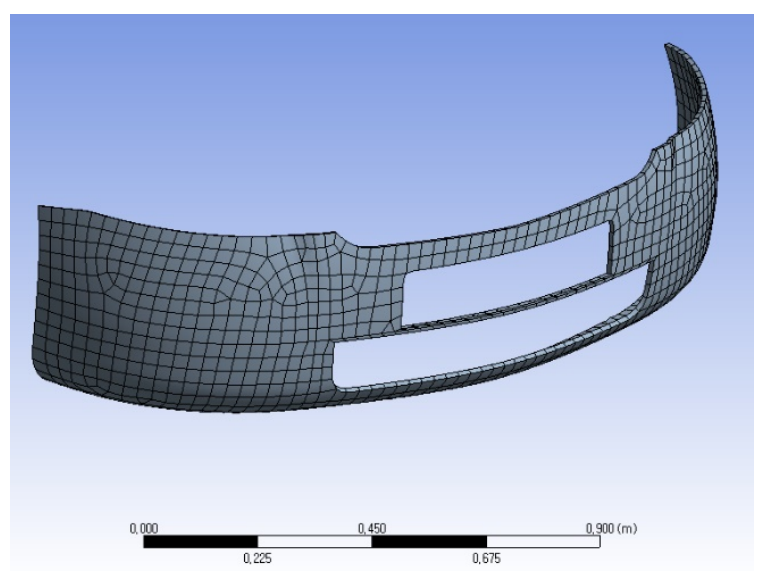

Figure.6. Finite element model for an automotive bumper

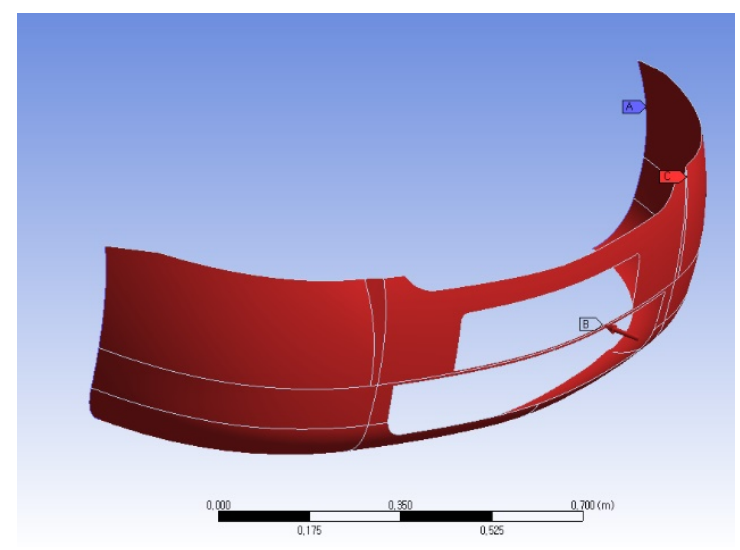

Figure.7. Boundary conditions for the finite element model

Input parameters for the response surface methodology were temperature, thickness of bumper, element size, and application of the vapor smoothing. The range of each parameter is shown in Table 1.

Table 1. The factor ranges for input parameters

\begin{tabular}{ll} 
Input parameter & {$[\mathrm{min}, \max ]$} \\
\hline Temperature $\left[{ }^{\circ} \mathrm{C}\right]$ & {$[-40,70]$} \\
Thickness of Bumper $[\mathrm{m}]$ & {$[0.01,0.03]$} \\
Element Size $[\mathrm{m}]$ & {$[0.03,0.04]$} \\
Vapor smoothing & $\mathrm{w} / \mathrm{o}, \mathrm{w} /$
\end{tabular}

Box-Behnken design was used for the response surface methodology. Design points were generated by using Minitab software as shown in Figure. 8. And the output parameter was a maximum of total deformation of the bumper.

\section{Results and Discussion}

The first describes thermo-mechanical properties of $\mathrm{ABS}$ specimens measured by DMA and dilatometry. The second subsection depicts numerical simulation for an automotive bumper made of ABS.

\subsection{Thermo-mechanical properties of ABS specimens}

Four different splits were measured, which were exposed to different amount of acetone vapor; $0 \mathrm{ml}, 5 \mathrm{ml}, 10 \mathrm{ml}$ and $15 \mathrm{ml}$. Three specimens per each split were fabricated and measured in the same condition. Averaged thermo-mechanical properties of the 


\section{S.U. Zhang / Advances in Science, Technology and Engineering Systems Journal Vol. 2, No. 6, 157-161 (2017)}

ABS specimens were obtained, which are the storage modulus and $\mathrm{CTE}$ as shown in Figure.9 and Figure.10.

\begin{tabular}{|c|c|c|c|c|c|c|c|c|}
\hline+ & C1 & C2 & C3 & c4 & C5 & c6 & $\begin{array}{cl}\text { C7 } & \\
\end{array}$ & C8-T \\
\hline & Stdorder & RunOrder & Ptтype & Blocks & Temperature[C] & Thickness of Bumper [m] & Element size [m] & Vapor smoothing \\
\hline 1 & 1 & 1 & 2 & 1 & -40 & 0.01 & & \\
\hline 2 & 2 & 2 & 2 & 1 & 70 & 0.01 & & w/o \\
\hline 3 & 3 & 3 & 2 & 1 & -40 & 0.03 & & w/o \\
\hline 4 & 4 & 4 & 2 & 1 & 70 & 0.03 & & w/o \\
\hline 5 & 5 & 5 & 2 & 1 & -40 & 0.02 & & w/o \\
\hline 6 & 6 & 6 & 2 & 1 & 70 & 0.02 & & w/o \\
\hline 7 & 7 & 7 & 2 & 1 & -40 & 0.02 & & w/o \\
\hline 8 & 8 & 8 & 2 & 1 & 70 & 0.02 & & w/o \\
\hline 9 & 9 & 9 & 2 & 1 & 15 & 0.01 & & w/o \\
\hline 10 & 10 & 10 & 2 & 1 & 15 & 0.03 & & w/o \\
\hline 11 & 11 & 11 & 2 & 1 & 15 & 0.01 & & w/o \\
\hline 12 & 12 & 12 & 2 & 1 & 15 & 0.03 & & w/o \\
\hline 13 & 13 & 13 & 0 & 1 & 15 & 0.02 & & w/o \\
\hline 14 & 14 & 14 & 0 & 1 & 15 & 0.02 & & $\mathrm{w} / \mathrm{o}$ \\
\hline 15 & 15 & 15 & 0 & 1 & 15 & 0.02 & & $w / o$ \\
\hline 16 & 16 & 16 & 2 & 1 & -40 & 0.01 & & $\mathrm{w} /$ \\
\hline 17 & 17 & 17 & 2 & 1 & 70 & 0.01 & & w/ \\
\hline 18 & 18 & 18 & 2 & 1 & -40 & 0.03 & & $\mathrm{w} /$ \\
\hline 19 & 19 & 19 & 2 & 1 & 70 & 0.03 & & $\mathrm{w} /$ \\
\hline 20 & 20 & 20 & 2 & 1 & -40 & 0.02 & & w/ \\
\hline 21 & 21 & 21 & 2 & 1 & 70 & 0.02 & & w/ \\
\hline 22 & 22 & 22 & 2 & 1 & -40 & 0.02 & & $\mathrm{w} /$ \\
\hline 23 & 23 & 23 & 2 & 1 & 70 & 0.02 & & w/ \\
\hline 24 & 24 & 24 & 2 & 1 & 15 & 0.01 & & w/ \\
\hline 25 & 25 & 25 & 2 & 1 & 15 & 0.03 & & w/ \\
\hline 26 & 26 & 26 & 2 & 1 & 15 & 0.01 & & w/ \\
\hline 27 & 27 & 27 & 2 & 1 & 15 & 0.03 & & \\
\hline 28 & 28 & 28 & 0 & 1 & 15 & 0.02 & & \\
\hline 29 & 29 & 29 & 0 & 1 & 15 & 0.02 & & \\
\hline 30 & 30 & 30 & 0 & 1 & 15 & 0.02 & 0.04 & \\
\hline
\end{tabular}

Figure.8. Design points generated by Minitab software

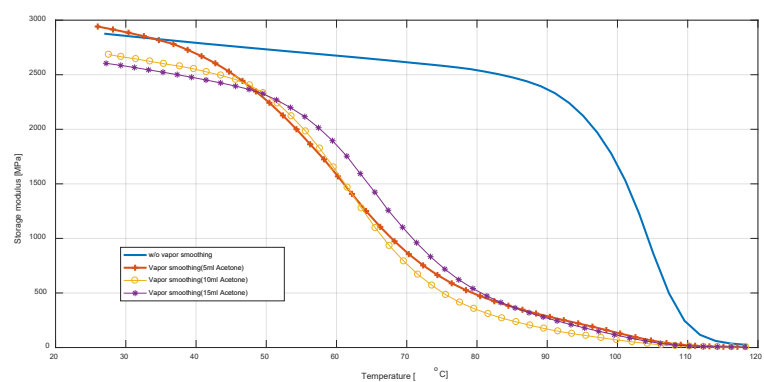

Figure.9. Average of temperature dependent storage moduli for four splits

Figure.9 graphically shows the difference between specimens without vapor smoothing process and ones with vapor smoothing process. The glass transition temperature of the specimens with the vapor smoothing process is much lower than one without the vapor smoothing process.

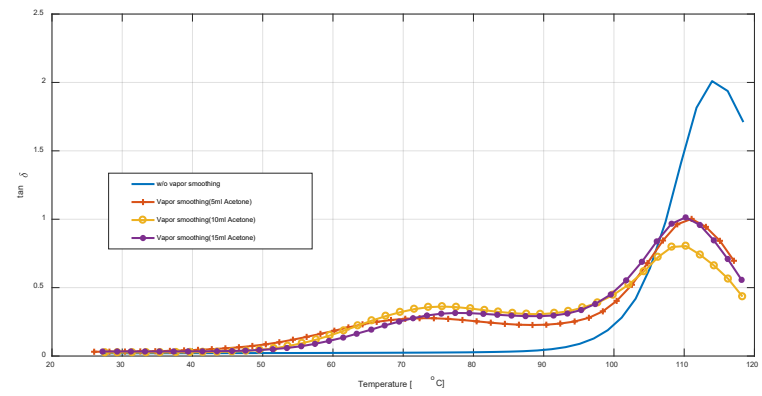

Figure.10. Average of $\tan \delta$ for four splits

Figure.10 graphically shows the difference between specimens without vapor smoothing process and ones with vapor smoothing process in terms of $\tan \delta$. In the graph of first split, peak point of $\tan \delta$ occurs at about $114^{\circ} \mathrm{C}$. However, the first peaks of the other specimens with the vapor smoothing are shown at $67^{\circ} \mathrm{C}, 71^{\circ} \mathrm{C}$, and $74^{\circ} \mathrm{C}$. Figure. 11 shows that CTEs vary as the temperature increases. CTE of the specimen without vapor smoothing is relatively constant.

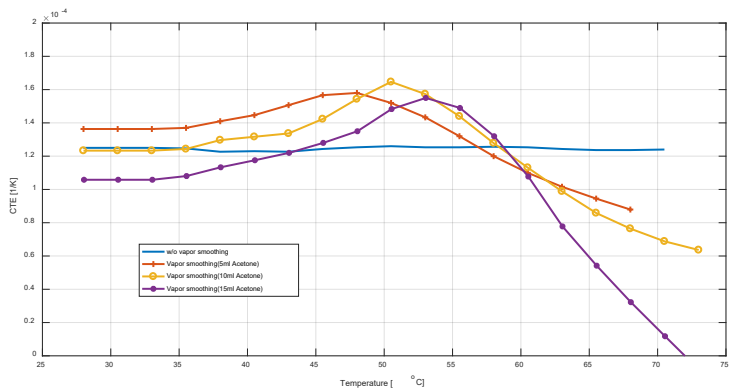

Figure.11. Average of the temperature dependent CTEs for four splits

However, CTEs of the others with vapor smoothing have large variations and have one peak between $45^{\circ} \mathrm{C}$ and $60^{\circ} \mathrm{C}$. As the temperature increases more than $60^{\circ} \mathrm{C}$, CTEs of the samples with vapor smoothing decreases.

Figure.9, Figure.10 and Figure. 11 obviously shows which split does not have the vapor smoothing process. Figures represent that the vapor smoothing process weakens thermal stability of ABS 3D printed parts. However, above figures could not show any correlation between CTE and the amount of acetone vapor in the range of $5 \mathrm{ml}$ to $15 \mathrm{ml}$.

\subsection{Numerical evaluation for an automotive bumper made of $A B S$}

The output parameter was the maximum value of total deformation of the automotive bumper as shown in Figure. 12. The maximum deformation occurs at the center of the bumper under the given boundary conditions.
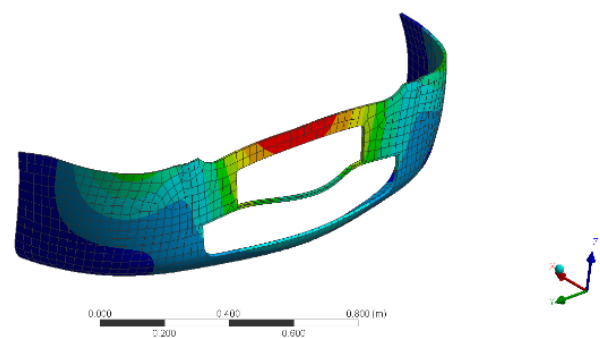

Figure.12. Total deformation of the automotive bumper

Using the response surface methodology and the finite element analysis, two regression equations with varying application of the vapor smoothing process were obtained as follow

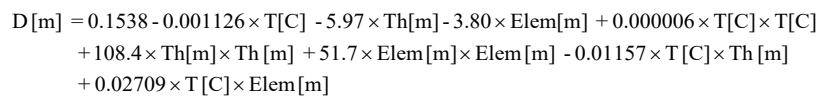

$\mathrm{D}[\mathrm{m}]=0.182-0.000793 \times \mathrm{T}[\mathrm{C}]-7.10 \times \mathrm{Th}[\mathrm{m}]-3.81 \times \operatorname{Elem}[\mathrm{m}]+0.000006 \times \mathrm{T}[\mathrm{C}] \times \mathrm{T}[\mathrm{C}]$ $+108.4 \times \mathrm{Th}[\mathrm{m}] \times \mathrm{Th}[\mathrm{m}]+51.7 \times$ Elem $[\mathrm{m}] \times \operatorname{Elem}[\mathrm{m}]-0.0116 \times \mathrm{T}[\mathrm{C}] \times \mathrm{Th}[\mathrm{m}]$ $+0.0271 \times \mathrm{T}[\mathrm{C}] \times \operatorname{Elem}[\mathrm{m}]+0.2 \times \mathrm{Th}[\mathrm{m}] \times \operatorname{Elem}[\mathrm{m}]$

where, $\mathrm{D}$ is the maximum deformation of the automotive bumper, $\mathrm{T}$ is the temperature, Th is the thickness of the bumper and Elem is the size of finite elements. When the vapor smoothing process is not applied, Equation (4) could be used. Otherwise, equation (5) could be used to estimate the maximum deformation of the bumper. R-square value of the response surface model was $81 \%$. Two equations represent that the maximum deformation is 


\section{S.U. Zhang / Advances in Science, Technology and Engineering Systems Journal Vol. 2, No. 6, 157-161 (2017)}

sensitive to the thickness of bumper and the temperature under given ranges. Based on two equations, contour plots were computed by using Minitab software as shown in Figure.12. According to the vapor smoothing process, two design guidelines could be obtained by using the response surface methodology, which might be useful information for bumper designers.

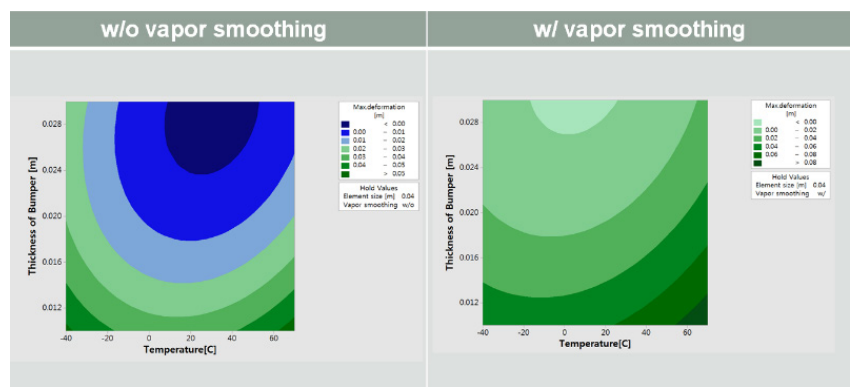

Figure.13. Contour plots according to the vapor smoothing process

\section{Conclusion}

In this study, the temperature-dependent mechanical properties of ABS parts were measured by used dynamic mechanical analyzer and dilatometry, which were fabricated by FDM and the vapor smoothing technique. The vapor smoothing process weakens thermal stability of ABS $3 \mathrm{D}$ printed parts. According to the temperature-dependent storage modulus of $3 \mathrm{D}$ printed $\mathrm{ABS}$ specimen, the glass transition temperature dramatically decreases by the process. The vapor smoothing process made relatively large variation in CTE of ABS 3D printed parts. Based on the measured thermos-mechanical properties of ABS specimen, finite element analysis and response surface methodology for an automotive bumper made of ABS were performed so that two design guidelines could be provided according to the vapor smoothing process.

\section{Acknowledgment}

This work was supported by Dong-Eui University Grant (201702720001) and the National Research Foundation of Korea (NRF) grant funded by the Korea government(MSIT) (No. NRF2017R1C1B5074219).

\section{References}

[1] Lyu MY, Choi TG. Research trends in polymer materials for use in lightweight vehicles 2015;16:213-20. doi:10.1007/s12541-015-0029-x.

[2] Gao W, Zhang Y, Ramanujan D, Ramani K, Chen Y, Williams CB, et al. The status, challenges, and future of additive manufacturing in engineering. Comput Des 2015;69:65-89. doi:10.1016/j.cad.2015.04.001.

[3] Chen L, He Y, Yang Y, Niu S, Ren H. The research status and development trend of additive manufacturing technology. Int $\mathrm{J}$ Adv Manuf Technol 2017;89:3651-60. doi:10.1007/s00170-016-9335-4.

[4] Kuo C-C, Mao R-C. Development of a Precision Surface Polishing System for Parts Fabricated by Fused Deposition Modeling. Mater Manuf Process 2016;31:1113-8. doi:10.1080/10426914.2015.1090594.

[5] Garg A, Bhattacharya A, Batish A. Chemical vapor treatment of ABS parts built by FDM: Analysis of surface finish and mechanical strength. Int J Adv Manuf Technol 2017;89:2175-91. doi:10.1007/s00170-016-9257-1.
[6] Singh R, Singh S, Singh IP, Fabbrocino F, Fraternali F. Investigation for surface finish improvement of FDM parts by vapor smoothing process. Compos Part B Eng 2017;111:228-34. doi:10.1016/j.compositesb.2016.11.062.

[7] Singh J, Singh R, Singh H. Repeatability of linear and radial dimension of ABS replicas fabricated by fused deposition modelling and chemical vapor smoothing process: A case study. Measurement 2016;94:5-11. doi:10.1016/j.measurement.2016.07.064.

[8] Cantrell JT, Rohde S, Damiani D, Gurnani R, DiSandro L, Anton J, et al. Experimental characterization of the mechanical properties of 3D-printed ABS and polycarbonate parts. Rapid Prototyp J 2017;23:811-24. doi:10.1108/RPJ-032016-0042.

[9] Gao H, Kaweesa D V., Moore J, Meisel NA. Investigating the Impact of Acetone Vapor Smoothing on the Strength and Elongation of Printed ABS Parts. Jom 2017;69:580-5. doi:10.1007/s11837-016-2214-5.

[10] Belter JT, Dollar AM. Strengthening of 3D printed fused deposition manufactured parts using the fill compositing technique. PLoS One 2015;10:1-19. doi:10.1371/journal.pone.0122915.

[11] Melenka GW, Schofield JS, Dawson MR, Carey JP. Evaluation of dimensional accuracy and material properties of the MakerBot 3D desktop printer. Rapid Prototyp J 2015;21:618-27. doi:10.1108/RPJ-09-2013-0093. 